\title{
Analysis on the Current Situation of Conflict Management and Future Prospects
}

\author{
Yujie Yi \\ School of Management, Jinan University, Guangzhou, China \\ Email:645476250@qq.com
}

How to cite this paper: Yi, Y.J. (2019) Analysis on the Current Situation of Conflict Management and Future Prospects. Open Journal of Business and Management, 7, 1053-1062. https://doi.org/10.4236/ojbm.2019.72071

Received: April 9, 2019

Accepted: April 25, 2019

Published: April 28, 2019

Copyright $\odot 2019$ by author(s) and Scientific Research Publishing Inc. This work is licensed under the Creative Commons Attribution International License (CC BY 4.0).

http://creativecommons.org/licenses/by/4.0/

\section{c) (i) Open Access}

\begin{abstract}
Inevitable conflicts in the workplace have dual effects on organizations. How to manage conflicts and make it play a constructive role in the organization is of great importance to the success of the organization. It is also one of the necessary management skills for managers. In order to enable managers to face up to conflicts, learn to manage conflicts in the right way, and play a constructive role in the organization. This paper studies conflict and conflict management behaviors by means of literature research. Firstly, this paper defines the meaning of conflict management and clarifies the specific content of conflict management. Then it reviews and analyses the research on conflict management by scholars at home and abroad. Finally, it points out the shortcomings of current research on conflict management, and briefly looks forward to the future development of conflict management research.
\end{abstract}

\section{Keywords \\ Conflict, Conflict Management}

\section{Preface}

According to a survey by American historian Slater, managers manage conflicts about $20 \%$ of their working time, and conflicts are inevitable in their work. People have different opinions about the conflict, which can be roughly divided into three stages. In the first stage, people think that conflict is harmful, synonymous with irrationality and destruction, and should be avoided in the organization. In the second stage, people realize that conflict is inevitable and we should accept and rationalize it. In the third stage, it is further recognized that conflicts can be constructive and that teams or organizations need to maintain a certain level of conflict. Throughout the researchers' researchs on the impact of conflict, scholars have also launched a series of studies. Ma Hongyu (2011) [1] 
pointed out that the relationship between organizational conflict and organizational performance is inverted U-shaped. Zhou Mingjian (2014) [2] research shows that team task conflict has a positive impact on team creativity, and relationship conflict has a negative impact on team creativity. Team effectiveness plays a mediating role in it. $\mathrm{Hu} \mathrm{N}$ et al. (2017) [3] argued that inter-organizational relationship conflicts were negatively correlated with organizational innovation performance. The relationship between task conflict and innovation performance is U-shaped. Hjerto K B and Kuvaas B (2017) [4] show that cognitive task conflict is negatively correlated with team performance, and emotional conflict is negatively correlated with team job satisfaction. Emotional task conflict is positively correlated with team performance. From the above research point of view, it can be said that conflicts in organizations are a double-edged sword. We cannot blindly think that they are harmful to avoid and ignore. What we need is to adopt the right way to manage and guide conflicts and make them play a constructive role. How to play the constructive role of conflict in organizations depends on how to manage conflict. This paper reviews the research on conflict management at home and abroad in terms of the meaning, influencing factors and results of conflict management, and points out the shortcomings of current research and prospects for future research.

\section{The Conceptual Connotation of Conflict Management}

Research on the division of conflict management methods is mostly based on the management grid theory of Blake and other dual concerns (caring for people and relationship production). Blake and Mouton (1968) [5] designed another conflict grid model based on the original "management grid" model and divided the conflict management into five types. $(1,1)$ Avoidance. Managers regard the possibility of avoiding conflicts as an effective way to relieve sudden emergencies, $(1,9)$ Moderation. It considers that differences between the two parties to a conflict can coexist peacefully by easing tension or maintaining superficial harmony, but in fact, the root causes of the conflict remain unresolved. $(9,1)$ Press. Most people would think that conflict can force both sides of the conflict to obey through power. $(5,5)$ Compliance. In this way, both sides of the conflict need to make submission or negotiation. As a result, no one wins or loses. It is a more practical way to solve the conflict. $(9,9)$ Face. In this way that the conflict problem can be solved by facing actively. It is a more active way of conflict management, which can thoroughly resolve conflicts. Thomas and Kilmann (1974) [6] based on the "management grid" theory and describe the conflict management behaviors in organizations and teams with five conflict management modes: Competition, cooperation, avoidance, accommodation, compromise, from the two dimensions of caring for themselves and others. Rahim (1983) [7] divided conflict management into five types: cooperation, Obliging, competition, avoidance and compromise from the perspective of caring for himself and others. Later this method has been widely used. Tjosvold et al. (2003 [8] classified con- 
flict management behaviors into three categories: integration, competition and avoidance. Chen Jing et al. (2006) [9] proposed conflict management in Chinese context. They classified Chinese interpersonal conflict management into three categories: coordination, concession and confrontation. Aw V K J and Ayoko O B (2017) [10] believed that problem solving, facing up to and informal behaviors can better and practically divide conflict management behavior in organizations. Among them, problem solving includes the way of cooperation and compromise divided by predecessors, the way of dealing with conflicts which is equivalent to competition, and the way of avoiding and obeying is informal behavior. The details are as follows (Table 1).

\section{The Factors Affecting Conflict Management}

This article will answer the following factors for the impact of conflict management, why should we manage conflicts? When is it managed? And how to manage conflicts in the workplace? Research on the influencing factors of conflict management mainly focuses on the individual level and organizational level.

\subsection{Individual Level}

The demographic characteristics of different individuals and individual psychological characteristics such as gender, role preference, stress, and emotional intelligence will affect the way individuals adopt different conflict management methods. Zhang S J et al. (2015) [11] found that Chinese intelligence companies have a significant positive correlation between emotional intelligence and conflict management methods of cooperation, compromise and competition. And the conflict management method of cooperation is significantly positively related to the innovation performance of enterprises. Yeung D Y et al. (2015) [12] had shown that the role and age of conflict participants in an organization has an important impact on how conflicts are managed. Older employees are more inclined to adopt conflict avoidance management methods when managing conflicts with superiors than younger employees. When managing conflicts with lower levels, younger employees use less conflict management methods. They also found that that the negative correlation between avoid conflict management method and interpersonal relationships is only found among young employees,

Table 1. Division of conflict management methods.

\begin{tabular}{cc}
\hline Author & Conflict management \\
\hline Blake and Mouton (1968) [5] & Avoid, Moderation, Press, Compliance, face \\
Thomas \&Kilmann (1974) [6] & Competition, cooperation, avoidance, accommodation, compromise \\
Rahim(1983) [7] & Cooperation, Obliging, competition, avoidance, compromise \\
Tjosvold, etc (2003) [8] & Cooperation, competition, avoidance \\
Jing Chen(2006) [9] & Coordination, concession, confrontation \\
Aw V K J, Ayoko O B (2017) [10] & Problems solving behavior, facing up to behavior, informal \\
& behavior \\
\hline
\end{tabular}


but not among older employees. Yang MY (2015) [13] studied the relation between behavior of Trait Emotion, Instantaneous Emotion and conflict framework, conflict management. The results of the study found that positive emotional traits and positive momentary emotions are related to the framework of compromise. The compromise framework will adopt cooperation management method. Another import factor is Leadership. Managers' different leadership styles will affect how they perceive conflicts and how they choose to solve them. Saeed T et al. (2015) [14] show that leaders who demonstrate a transformational style are more inclined to adopt integrated and avoid conflict management methods at work. Managers who show more trading styles have chosen compromise management method. Managers who are considered to have a laissez-faire leadership style adopt a style of avoidance to manage conflicts with subordinates. Jit $\mathrm{R}$ et al. (2016) [15] show that in the workplace, service leaders tend to be persuasive, patient, modest and choose employee-centric approach to resolve conflict, regardless of whether they play the role of a third-party mediator/arbitrator or directly face conflicts with subordinates. This kind of conflict management method can bring a fair and tolerant organizational atmosphere to the organization and facilitate the formation of organizational citizenship behavior.

\subsection{Organizational Factors}

The factors affecting conflict management at the organizational level mainly focus on three aspects: organizational level, organizational culture, and organizational fairness.

There are great differences in conflict management among employees of different levels or working years in organizations. Blake and Mouton (1968) [5] studied the conflict management styles of eight different levels in the organization, from the bottom up to the grassroots supervisor and chairman of the board of directors. The results show that managers with higher levels are more inclined to adopt conflict management methods of competition and cooperation, and employees at lower levels are more inclined to adopt conflict management methods of compliance, compromise and avoidance. Further research by Thomas $\mathrm{K}$ W et al. (2008) [6] at the organizational level shows that as organizational levels increase, conflict management for dictatorial (competition and cooperation) increases, and conflict management for moderation (avoidance and relocation) decreases. The compromised conflict management model has a curvilinear relationship with the organizational hierarchy, and conflict management at the highest and lowest organizational levels is reduced. Cultural background, cultural environment, cultural system, values and so on are all important antecedents that influence the way conflicts are managed. The study by Komarraju et al. (2008) [16] examines the role of horizontal and vertical individualism and collectivism in the choice of conflict management models. Studies have shown that an individualistic-oriented culture emphasizes the need to meet individual needs. Individuals under the influence of this culture are more inclined to adopt 
mandatory or dominant conflict management methods than altruistic or evasive conflict management methods. Collectivist culture-oriented individuals are more likely to choose integrated conflict management method for the collective interests to sacrifice individual interests. And the study further pointed out that horizontal collectivism is more likely to prefer altruistic methods, while vertical collectivists prefer to avoid conflict management methods. Bélanger $\mathrm{J} J$ et al. (2015) [17] studied the relationship between supervisors and subordinates' choice of conflict management methods for subordinates. Studies have shown that when supervisors take more stringent rights to employees who need high-closed cognition, Employees are more inclined to adopt constructive (solving problem) conflict management methods. When the supervisor takes a more moderate right to the employees who need lower closed cognition, Employees are more inclined to adopt a constructive approach to conflict management. Yang M Y (2015) [13] found that the team's positive trait emotions and positive short-term emotions are positively related to cooperative conflict management. Tatum and Eberlin (2008) [18] examine the relationship between organizational justice and conflict management. The empirical results show that managers who are sensitive to organizational justice issues are more likely to adopt cooperative conflict management methods in the face of conflicts with employees. Managers who are insensitive to the issue of organizational justice tend to seek competitive or dominant conflict resolution strategies in a potentially high conflict situation.

In summary, the existing research on the factors affecting conflict management mainly focuses on the research perspectives of organizational factors, leadership factors and individual factors. Among them, Organizational factors and leadership factors summarize the causes of conflict management from people's perception of the surrounding environment. Individual factors show that conflict management needs to start with the differences of psychological processes among individuals can interact and influence each other.

\section{The Study of the Outcome Variables of Conflict Management Methods}

There are relatively few research results on the outcome variables of conflict management methods. This paper mainly elaborates from the organizational level and individual level

\subsection{Individual Level}

The impact of different conflict management methods on the individual level of employees is mainly focused on the specific organizational behavior and psychological state of the employees. Yang I et al. (2017) [19] take the leadership of Chinese organizations as the research object. The research shows that there is a positive correlation between the conflict management methods of leadership avoidance in the organization and the subordinate's sense of fairness, trust and 
happiness. Such a result is closely related to the distance between rights in China's collectivist culture and organizations. Yu Jingjing et al. (2015) [20] used the employees of Shandong enterprises as the survey object. The research results show that the conflict management method of competition and cooperation has a significant positive impact on the behavior of employees, and psychological security plays a part of intermediary role between cooperative conflict management and voice behavior. Later, they using the questionnaire survey method, 430 employees from more than 30 enterprises in Henan, Shandong, Jiangsu, Shanghai and other provinces were used as research objects, and the hierarchical regression method was used for data analysis. The study found that cooperative, competitive and avoiding conflict management methods have a significant positive impact on employee innovation behavior. Team learning plays a moderating role. Le Yun et al. (2014) [21] studied the relationship between the three different conflict management methods of the project manager's decision, prudence, and avoidance, and the employee's turnover behavior. The results show that adopting a decisive and prudent conflict management method will significantly enhance the quality of LMX, while the avoidance method will lead to the low quality of LMX and thus enhance the employee turnover intention. LMX partially mediates between conflict patterns and turnover intention and the experience of working together will strengthen the impact of conflict management method on LMX.

\subsection{Organizational Level}

At the organizational level: Aw V K J and Ayoko O B (2017) [10] studies show that when subordinates adopt constructive conflict management methods (cooperation, compromise), they can enhance the quality of team members' exchange relationship and then improve the performance of the organization. Constructive conflict management is positively related to transformational leadership Improve the quality of organizational members' exchange relationships and improve organizational performance. Constructive conflict management methods are positively related to transformational leadership. Le Yun et al. (2014) [21] show that decisive and prudent conflict management methods will improve the quality of LMX, while avoidance will reduce the quality of LMX. An empirical study by Zhang S J et al. (2015) [11] shows that cooperative conflict management method is significantly positively correlated with organizational innovation performance. Chen Xiaohong and Zhao Ke (2010) [22] found that conflict and conflict management behavior has significant impact on team performance through empirical research on conflicts, conflict management behaviors and team performance of 76 work teams. The competitive conflict management method will increase the relationship conflict, which leads to the decrease of team performance. The avoidance conflict management method is negatively correlated with the relationship conflict.

In summary, we know that conflict is inevitable, and it will have an impact on 
the organizations and individuals. Not only will it affect the specific behavior of employees, the psychological state of employees, or even the performance and innovation of organizations. Mastering the methods of conflicts management is very important in the workplace, especially for managers, which is also a very big challenge.

\section{Future Research Prospects}

As an important function of the organization, conflict management has been paid attention to by psychology and organizational behavior scholars for decades. The existing literature has made important research progress on the concept of conflict management, the study of antecedent variables and outcome variables. However, with the development of the times, conflict management research still needs to further expand its research scope. On the basis of further summarizing the shortcomings of previous studies, it is necessary to further strengthen the in-depth study on the following aspects of conflict management.

\subsection{Strengthening Cross-Cultural Conflict Management Research}

Current research on conflict management is either based on Eastern cultural contexts or based on Western cultural contexts, and research on cross-cultural contexts is inadequate. For example, the relative power distance can influence how employees perceive the leader's conflict management intervention. The power distance is relatively low in Western countries such as the Netherlands, and relatively high in Eastern countries such as China. The compulsory conflict management behavior in countries with high power distance may be more acceptable, while in low-power distance countries, compulsory conflict management Behaviors are more easily perceived by employees as dictatorship conflict management.

\subsection{Deepening Research on the Impact Mechanism of Conflict Management}

Firstly, it is necessary to further explore the mechanism of conflict management on outcome variables. Current research focuses on the impact of different conflict management methods on organizational performance, organizational innovation, organizational justice and employee sentiment, and impact on leaders themselves and individual factors of employees. For example, Zhang (2011) [23] verified the mediating effect of conflict management on transformational leadership and team performance, and pointed out that the relationship between transformational leadership and potential of conflict management could be further studied in the future. Secondly, future research can consider revealing the influencing factors and results of conflict management from different theoretical perspectives. Secondly, future research can consider revealing the influencing factors and results of conflict management from different theoretical perspectives. In the future, we can further expand other theoretical perspectives for 
multi-angle research, such as the use of social learning theory to reveal the impact mechanism of conflict management. Because conflict management can create a better organizational and social environment for employees to learn, it is conducive to the improvement of individual capabilities and career development.

\subsection{Exploring the Conflict Management of Employment Relationship}

At present, the research on conflict management is mostly limited to the conflict management between teams and the conflict management between leaders and subordinates, which has not contributed enough to the conflict management between employees and organizations.

At present, China's social structure has undergone profound changes, and industrial relations have become one of the basic relations of Chinese society. Chang kai (2005) [24] believes that China has begun to enter a period of frequent labor conflicts and conflicts. Labor conflicts and labor conflicts have exerted far-reaching influence on China's economy and society, increasing economic costs and causing losses to enterprises. Labor-capital conflict and labor-capital conflict have become important factors affecting social harmony and stability. At present, whether the management of labor conflicts and collective actions in China is proper or not has considerable political influence. Therefore, it is of theoretical and practical significance to study the conflict management mode of employment relationship.

\section{Conflicts of Interest}

The author declares no conflicts of interest regarding the publication of this paper.

\section{References}

[1] Ma, H. (2011) A Conflict Management Strategy Based on the Inverted "U" Relationship between Conflict and Organizational Performance. Human Resource Development in China, 3, 15-19.

[2] Zhou, M.J., Pan, H.B. and Ren, J.F. (2014) Team Conflict and Team Creativity: The Mediating Role of Team Potency. Management Review, 26, 120-130.

[3] Hu, N., Chen, Z., Gu, J.B., Huang, S.L. and Liu, H.F. (2017) Conflict and Creativity in Inter-Organizational Teams: The Moderating Role of Shared Leadership. International Journal of Conflict Management, 28, 74-102.

[4] Hjerto, K.B. and Kuvaas, B. (2017) Burning Hearts in Conflict: New Perspectives on the Intragroup Conflict and Team Effectiveness Relationship. International Journal of Conflict Management, 28, 50-73. https://doi.org/10.1108/ijcma-02-2016-0009

[5] Blake, R.R. and Mouton, J.S. (1968) The Managerial Grid: Key Orientations for Achieving Production Through People. Gulf Publishing Company, Houston.

[6] Thomas, K.W., Thomas, G.F. and Schaubhut, N. (2008) Conflict Styles of Men and Women at Six Organization Levels. International Journal of Conflict Management, 19, 148-166. https://doi.org/10.1108/10444060810856085 
[7] Rahim, M.A. (1983) A Measure of Styles of Handling Interpersonal Conflict. Academy of Management Journal, 26, 368-376.

[8] Tjosvold, D., Hui, C. and Yu, Z. (2003) Conflict Management and Task Reflexivity For Team In-Role and Extra-Role Performance in China. International Journal of Conflict Management, 14, 141-163. https://doi.org/10.1108/eb022895

[9] Chen, J., Chen, L. and Zhang, J. (2006) A Scale Study of Work Conflict Handling Styles between Supervisors and Subordinates. Psychological Science, 29, 926-928.

[10] Aw, V.K.J. and Ayoko, O.B. (2017) The Impact of Followers' Conflict Behaviors on Teams' Transformational Leadership, Team Member Exchange and Engagement. International Journal of Conflict Management, 28, 509-532. https://doi.org/10.1108/ijcma-04-2016-0020

[11] Zhang, S.J., Chen, Y.Q. and Hui, S. (2015) Emotional Intelligence, Conflict Management Styles, and Innovation Performance: An Empirical Study of Chinese Employees. International Journal of Conflict Management, 26, 450-478. https://doi.org/10.1108/ijcma-06-2014-0039

[12] Yeung, D.Y., Fung, H.H. and Chan, D. (2015) Managing Conflict at Work: Comparison between Younger and Older Managerial Employees. International Journal of Conflict Management, 26, 342-364. https://doi.org/10.1108/ijcma-06-2014-0044

[13] Yang, M.Y., Cheng, F.C. and Chuang, A. (2015) The Role of Affects in Conflict Frames and Conflict Management. International Journal of Conflict Management, 26, 427-449. https://doi.org/10.1108/ijcma-09-2013-0077

[14] Saeed, T., Almas, S., Anis-ul-Haq, M. and Niazi, G.S.K. (2014) Leadership Styles: Relationship with Conflict Management Styles. International Journal of Conflict Management, 25, 214-225. https://doi.org/10.1108/ijcma-12-2012-0091

[15] Jit, R., Sharma, C.S. and Kawatra, M. (2016) Servant Leadership and Conflict Resolution: A Qualitative Study. International Journal of Conflict Management, 27, 591-612. https://doi.org/10.1108/ijcma-12-2015-0086

[16] Komarraju, M., Dollinger, S.J. and Lovell, J.L. (2008) Individualism-Collectivism in Horizontal and Vertical Directions as Predictors of Conflict Management Styles. International Journal of Conflict Management, 19, 20-35. https://doi.org/10.1108/10444060810849164

[17] Bélanger, J.J., et al. (2015) Handling Conflict at Work: The Role of Fit between Subordinates' Need for Closure and Supervisors' Power Tactics. International Journal of Conflict Management, 26, 25-43. https://doi.org/10.1108/ijcma-09-2013-0083

[18] Tatum, B.C. and Eberlin, R.J. (2008) The Relationship between Organizational Justice and Conflict Style. Business Strategy, 9, 297-305. https://doi.org/10.1108/17515630810923603

[19] Yang, I. and Li, M. (2017) Can Absent Leadership Be Positive in Team Conflicts? : An Examination of Leaders' Avoidance Behavior in China. International Journal of Conflict Management, 28, 146-165. https://doi.org/10.1108/ijcma-12-2015-0083

[20] Yu, J.J., Jiang, S.F. and Zhao, S.M. (2015) A Study on the Relationship Between Conflict Management and Employee Voice Behavior-From the Perspective of Psychological Security and Power Distance. East China Economic Management, 29, 168-174.

[21] Le, Y., Bai, J. and Wang, S.W. (2014) The Role of Project Manager Conflict Management Model on Subordinates' Turnover Tendency. Journal of Tongji University (Natural Science Edition), 42, 1776-1782.

[22] Chen, X.H. and Zhao, K. (2010) An Empirical Study on the Relationship between 
Team Conflict, Conflict Management and Performance. Nankai Management Review, 13, 31-35.

[23] Zhang, X., Cao, Q. and Tjosvold, D. (2011) Linking Transformational Leadership and Team Performance: A Conflict Management Approach. Journal of Management Studies, 48, 1586-1611.https://doi.org/10.1111/j.1467-6486.2010.00974.x

[24] (2005) Changkai: Some Thoughts on the Legislation of the Right to Strike, Xuehai, No. 4. 